\section{Nível de hemoglobina entre idosos e sua associação com indicadores do estado nutricional e uso de serviços de saúde: Projeto Bambuí}

\author{
Hemoglobin level in older adults and the association \\ with nutritional status and use of health services: \\ the Bambuí Project
}

\begin{abstract}
1 Núcleo de Estudos em Saúde Pública e

Envelhecimento, Fundação Oswaldo Cruz/Universidade Federal de Minas Gerais, Belo Horizonte, Brasil.

Centro de Pesquisas René Rachou, Fundação Oswaldo Cruz, Belo Horizonte, Brasil. 3 Escola de Enfermagem, Universidade Federal de Minas Gerais, Belo Horizonte, Brasil.

Correspondência C. L. A. Silva

Núcleo de Estudos em Saúde Pública e Envelhecimento, Fundação Oswaldo Cruzl Universidade Federal de Minas Gerais. Av. Augusto de Lima 1715,

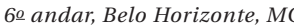
30190-002, Brasil.

claricelas@cpqrr.fiocruz.br

This study aimed to describe the prevalence of anemia and to evaluate the association between hemoglobin $(\mathrm{Hb})$ level and indicators of nutritional status and health services utilization in an elderly population ( $\geq 60$ years). Of the 1,742 older adults in the baseline of the Bambui cohort, 1,441 (82.7\%) were included. The dependent variable was Hb level, and the independent variables were number of hospitalizations and physician visits in the previous 12 months, body mass index (BMI), and serum albumin. Multivariate linear regression was performed, stratified by gender and adjusted for confounding factors. Prevalence of anemia was low (4.5\%), with no difference by gender, and higher in the oldest old (10.2\%). Low Hb level was associated with more physician visits and lower serum albumin and BMI in both men and women, suggesting a complex cycle between low Hb level, malnutrition, and medical care that should be considered when structuring health programs for the elderly.
\end{abstract}

Aging; Hemoglobins; Anemia; Nutritional Status; Health Services
Clarice Lima Álvares da Silva 1,2 Maria Fernanda Lima-Costa 1 Josélia Oliveira Araújo Firmo 1 Sérgio Viana Peixoto 1,3

\section{Introdução}

Os níveis de hemoglobina $(\mathrm{Hb})$ tendem a diminuir com o aumento da idade, pois a modulação hematopoiética se torna desbalanceada em decorrência da menor habilidade da medula óssea para responder a estímulos adversos, tais como sangramentos, infecções e danos citotóxicos 1,2. A anemia é, então, considerada um problema de saúde nessa população ${ }^{3,4}$, sendo elevada a sua prevalência após os 60 anos e ainda maior entre aqueles com 80 anos ou mais 5,6,7,8.

Baixos níveis de $\mathrm{Hb}$, mesmo entre idosos não anêmicos, têm sido relacionados a déficits de concentração e força muscular, baixo desempenho físico, dependência funcional e aumento da mortalidade por outras doenças 3,9,10,11. Embora não seja bem estabelecida a relação de causalidade, tem sido postulado que tal condição atua negativamente na saúde geral do idoso, agravando problemas preexistentes e levando ao surgimento de novas complicações 4 .

Pesquisas de base populacional entre idosos para determinar a prevalência de anemia e os fatores associados ao nível de $\mathrm{Hb}$ são escassas 12, sobretudo no Brasil. Os estudos nacionais incluem dois, desenvolvidos nos estados de Minas Gerais 13 e Pernambuco 14, com amostras de idosos cadastrados no Programa Saúde da Família (PSF); outro, da Região Sul do país 1, com amostra não probabilística de idosos saudáveis partici- 
pantes de grupos sociais; e apenas um de base populacional, desenvolvido em São Paulo 15 Apesar da escassez, os resultados dessas investigações podem ser considerados pioneiros no levantamento da anemia verificada na população idosa brasileira, com prevalências dessa condição variando entre $4 \%$ e $12 \%$ nas populações estudadas.

A associação entre baixos níveis de $\mathrm{Hb}$ e maior procura por serviços de saúde já foi bem estabelecida entre idosos usuários de sistemas de saúde públicos e privados americanos, mostrando impacto importante sobre seus custos 16 . Por outro lado, a sua associação com desnutrição proteico-calórica não tem sido muito explorada na literatura 17,18 , especialmente entre idosos residentes em comunidade. Entre idosos hospitalizados, a anemia é fortemente associada a menores valores de índice de massa corporal e albumina sérica 8,18,19,20. Portanto, parece haver forte relação entre níveis de $\mathrm{Hb}$, estado nutricional e uso de serviços de saúde em idosos, que deve ser mais bem explorada em estudos de base populacional, de forma a possibilitar uma adequada avaliação e elaboração de estratégias voltadas à saúde deste grupo.

No presente trabalho, foram utilizados dados da linha de base da coorte de Bambuí 21, Minas Gerais, Brasil, para descrever a prevalência de anemia e verificar a associação de nível de $\mathrm{Hb}$ com indicadores do estado nutricional e uso de serviços de saúde nessa população.

\section{Material e métodos}

\section{Coorte de idosos de Bambuí}

A coorte de idosos de Bambuí foi conduzida no Município de Bambuí, o qual conta com aproximadamente 15 mil habitantes, estando situado no Estado de Minas Gerais. Os participantes da linha de base foram identificados por meio de um censo completo realizado na cidade em 1996. Todos os 1.742 residentes na área urbana com 60 anos ou mais de idade, em 1o de janeiro de 1997, foram elegíveis para o estudo de coorte. Destes, $1.496(85,9 \%)$ realizaram medidas antropométricas e exames hematológicos, dos quais 55 foram excluídos da análise porque estavam em uso de suplementos nutricionais de ferro, vitamina $\mathrm{B}_{12} \mathrm{e} / \mathrm{ou}$ ácido fólico. Dessa forma $1.441(82,7 \%)$ idosos foram selecionados para o presente trabalho. O delineamento da coorte e os procedimentos adotados foram descritos em publicação anterior 21 .

A coorte de Bambuí foi aprovada pelo Comitê de Ética da Fundação Oswaldo Cruz, Rio de
Janeiro, e todos os participantes assinaram um termo de consentimento livre e esclarecido.

\section{Coleta de dados}

As informações para este trabalho foram obtidas por meio do questionário BHAS (Bambuí Health and Aging Study). As entrevistas foram realizadas na residência do participante e respondidas pelo idoso, exceto quando este estava impossibilitado em decorrência de déficit cognitivo ou de algum problema de saúde; neste caso, utilizou-se um respondente próximo.

A avaliação antropométrica (peso e altura) e a coleta de amostras de sangue para análises bioquímicas foram realizadas na clínica de campo do projeto (Posto Emanuel Dias). A avaliação antropométrica, feita por técnicos certificados após treinamento, empregou técnicas e equipamentos padrões (CMS Weighing Equipment Ltd. Londres, Reino Unido), estando os participantes vestidos com roupas leves e sem sapatos. Para a coleta de sangue, os participantes foram orientados a realizar jejum mínimo de 12 horas. Na impossibilidade de o voluntário se locomover até o posto, a coleta foi feita em seu domicílio. A dosagem do nível de $\mathrm{Hb}$ foi realizada mediante contador eletrônico (Coulter Counter T-890, Estados Unidos), e os níveis séricos de albumina e creatinina foram determinados por métodos enzimáticos padronizados, usando-se equipamento automático (Eclipse Vitalab; Merck, Holanda).

\section{Variáveis do estudo}

O nível de $\mathrm{Hb}(\mathrm{g} / \mathrm{dL}$ ) foi considerado como variável contínua, sendo a anemia caracterizada por valores abaixo de $13 \mathrm{~g} / \mathrm{dL}$, para o sexo masculino, e $12 \mathrm{~g} / \mathrm{dL}$, para feminino 22 . Idosos anêmicos foram classificados como casos graves quando apresentaram níveis de $\mathrm{Hb}$ abaixo de $10 \mathrm{~g} / \mathrm{dL}$ (para ambos os sexos) ou moderados quando apresentaram níveis de $\mathrm{Hb}$ entre $10 \mathrm{e}$ $12 \mathrm{~g} / \mathrm{dL}$, para o sexo feminino, e 10 a $13 \mathrm{~g} / \mathrm{dL}$, para o masculino ${ }^{5}$.

Outras variáveis incluídas no estudo foram: características sociodemográficas (sexo, idade e número de anos completos de escolaridade), história de diagnóstico médico para doenças (diabetes mellitus, hipertensão arterial e artrite), número de medicamentos consumidos nos últimos noventa dias, número de consultas médicas e de hospitalizações nos últimos 12 meses, tabagismo atual (entre os que haviam fumado pelo menos 100 cigarros ao longo da vida), níveis séricos de albumina $(\mathrm{mg} / \mathrm{dL})$, níveis séricos de creatinina $(\mathrm{g} / \mathrm{dL})$ e índice de massa corporal (IMC) (definido como a razão entre o peso e o 
quadrado da altura $-\mathrm{kg} / \mathrm{m}^{2}$ ). Níveis de albumina foram considerados baixos quando menores que $3,5 \mathrm{~g} / \mathrm{dL}$. O idoso foi classificado como baixo peso quando apresentou IMC abaixo de $18,5 \mathrm{~kg} / \mathrm{m}^{2} \mathrm{e}$ sobrepeso quando apresentou IMC acima de $24,99 \mathrm{~kg} / \mathrm{m}^{2} 23$.

\section{Análise dos dados}

Inicialmente, realizou-se a descrição de todas as variáveis por sexo, por intermédio do teste do qui-quadrado de Pearson e o teste t de Student, para comparação entre frequências e médias, respectivamente. A análise de variância, complementada com teste de Bonferroni, foi utilizada para comparação do nível de $\mathrm{Hb}$ entre as faixas etárias.

A análise multivariada foi realizada pela regressão linear múltipla, estimando-se os coeficientes da regressão $(\beta)$ e respectivos intervalos de $95 \%$ de confiança (IC95\%), para avaliar a associação entre nível de Hb e as variáveis: número de consultas médicas e de hospitalizações nos 12 meses precedentes à entrevista, IMC e níveis séricos de albumina. A análise foi ajustada por idade, escolaridade, tabagismo, diabetes, hipertensão, artrite, níveis séricos de creatinina e número de medicamentos consumidos, descritos na literatura como potenciais determinantes da redução dos níveis de $\mathrm{Hb}$ em idosos 24,25,26,27,28.

Todas as análises foram estratificadas por sexo e realizadas valendo-se do pacote estatístico Stata 10.0 (Stata Corp., College Station, Estados Unidos).

\section{Resultados}

Entre os 1.441 idosos incluídos neste estudo, 561 (38,9\%) eram homens e 880 (61,1\%), mulheres. A Tabela 1 apresenta as características da população estudada. A idade dos participantes variou entre 60 e 95 anos, sendo a média igual 69,1 anos (desvio padrão $=7,2$ anos), predominando a baixa escolaridade $(64,7 \%$ tinham menos de quatro anos de estudo). O tabagismo atual foi relatado por $18 \%$ dos participantes. A hipertensão arterial autorreferida foi a doença mais prevalente (56,8\%), e apenas $13,4 \%$ dos idosos não relataram uso de medicamentos nos últimos três meses.

\section{Tabela 1}

Características dos participantes do estudo, segundo sexo. Projeto Bambuí, Minas Gerais, Brasil, 1997.

\begin{tabular}{|c|c|c|c|c|}
\hline \multirow[t]{2}{*}{ Variáveis } & \multirow{2}{*}{$\begin{array}{c}\text { Total } \\
(\mathrm{N}=1.441)\end{array}$} & \multicolumn{2}{|c|}{ Sexo } & \multirow[t]{2}{*}{ Valor de $p$} \\
\hline & & $\begin{array}{l}\text { Homens } \\
(n=561)\end{array}$ & $\begin{array}{l}\text { Mulheres } \\
(\mathrm{n}=880)\end{array}$ & \\
\hline Idade em anos, média (DP) & $69,1(7,20)$ & $68,9(7,20)$ & $69,3(7,20)$ & 0,223 \\
\hline Escolaridade $<4$ anos (\%) & 64,7 & 61,5 & 66,7 & 0,046 \\
\hline Tabagismo atual (\%) & 18,0 & 30,1 & 10,2 & $<0,001$ \\
\hline \multicolumn{5}{|l|}{ História de diagnóstico médico (\%) } \\
\hline Diabetes & 11,7 & 9,1 & 13,3 & 0,015 \\
\hline Hipertensão & 56,8 & 47,2 & 62,8 & $<0,001$ \\
\hline Artrite & 31,3 & 26,4 & 34,5 & 0,001 \\
\hline \multicolumn{5}{|l|}{ Número de medicamentos consumidos nos últimos 90 dias (\%) } \\
\hline 0 & 13,4 & 21,8 & 8,1 & $<0,001$ \\
\hline $1-4$ & 60,9 & 63,5 & 59,2 & \\
\hline$\geq 5$ & 25,7 & 14,8 & 32,7 & \\
\hline Ocorrência de uma hospitalização ou mais nos últimos 12 meses (\%) & 21,9 & 17,8 & 24,4 & 0,003 \\
\hline \multicolumn{5}{|l|}{ Número de consultas médicas nos últimos 12 meses (\%) } \\
\hline 0 & 19,4 & 28,4 & 13,7 & $<0,001$ \\
\hline $1-3$ & 49,7 & 49,4 & 49,9 & \\
\hline$\geq 4$ & 30,9 & 22,3 & 36,4 & \\
\hline Creatinina (g/dL), média (DP) & $0,90(0,30)$ & $1,03(0,39)$ & $0,81(0,18)$ & $<0,001$ \\
\hline Albumina (mg/dL), média (DP) & $4,56(0,54)$ & $4,61(0,53)$ & $4,53(0,53)$ & 0,008 \\
\hline Índice de massa corporal (kg/m²), média (DP) & $25,11(4,96)$ & $23,93(4,21)$ & $25,86(5,26)$ & $<0,001$ \\
\hline Hemoglobina (g/dL), média (DP) & $14,5(1,40)$ & $15,3(1,40)$ & $13,9(1,10)$ & $<0,001$ \\
\hline
\end{tabular}

DP: desvio-padrão. 
Vinte e um vírgula nove por cento relataram pelo menos uma hospitalização e $80,6 \%$ uma consulta, no mínimo, com médico no último ano. O nível médio de Hb na população idosa de Bambuí foi igual a 14,5g/dL (desvio-padrão = 1,4). Com exceção da idade, as demais variáveis apresentaram diferenças significativas entre os sexos: os homens tinham maior escolaridade, maior prevalência de tabagismo e maiores níveis de creatinina, albumina e hemoglobina.

A Figura 1 descreve o nível de hemoglobina na população estudada, segundo sexo. A curva de distribuição da $\mathrm{Hb}$ entre os homens apresenta-se deslocada para a direita, refletindo o maior nível médio observado nesse grupo, em comparação ao sexo feminino. Já a distribuição do nível médio de $\mathrm{Hb}$ por faixa etária mostra uma redução desses níveis com o aumento da idade em ambos os sexos, de forma que idosos mais velhos ( $\geq$ 80 anos) apresentaram níveis significativamente menores do que as duas faixas etárias mais jovens, tanto entre homens quanto entre mulheres (Figura 2).

A prevalência de anemia na coorte foi de $4,5 \%$ ( $\mathrm{n}=65)$, sendo $4,8 \%(\mathrm{n}=27)$ no sexo masculino e $4,3 \%(n=38)$ no feminino $(p=0,659)$. Quando avaliada entre os mais velhos ( $\geq 80$ anos), os valores foram significativamente maiores em comparação com os dos mais jovens, alcançando 10,2\% $(n=15)$ deste grupo $(\mathrm{p}<0,0001)$ (homens: $8,9 \%$; $\mathrm{n}=5$ e mulheres: $11 \% ; \mathrm{n}=10 ; \mathrm{p}=0,689$ ). Apenas $0,21 \%(n=3)$ dos idosos tinha anemia grave, sem diferença entre os sexos $(p=0,842)$. A anemia moderada foi mais prevalente, atingindo $3,86 \%$ ( $n=62)$ da população, mas igualmente sem diferença entre os sexos $(p=0,246)$.

A Tabela 2 mostra a distribuição do nível médio de $\mathrm{Hb}$ de acordo com indicadores do uso de serviços de saúde e do estado nutricional dos idosos. Para o sexo masculino, o nível médio de $\mathrm{Hb}$ foi significativamente menor entre aqueles com maior número de consultas médicas no último ano, baixos níveis de albumina e desnutrição/ eutrofia. Para o sexo feminino, menores níveis médios de $\mathrm{Hb}$ foram observados entre as idosas com história de hospitalização no último ano e baixos níveis de albumina.

Os resultados da regressão linear múltipla para o estudo da associação entre os níveis de $\mathrm{Hb}$ e os indicadores do uso de serviços de saúde e do estado nutricional estão apresentados na Tabela 3. Em ambos os sexos, o relato de quatro ou

Figura 1

Distribuição dos níveis de hemoglobina entre idosos, segundo sexo. Projeto Bambuí, Minas Gerais, Brasil, 1997.
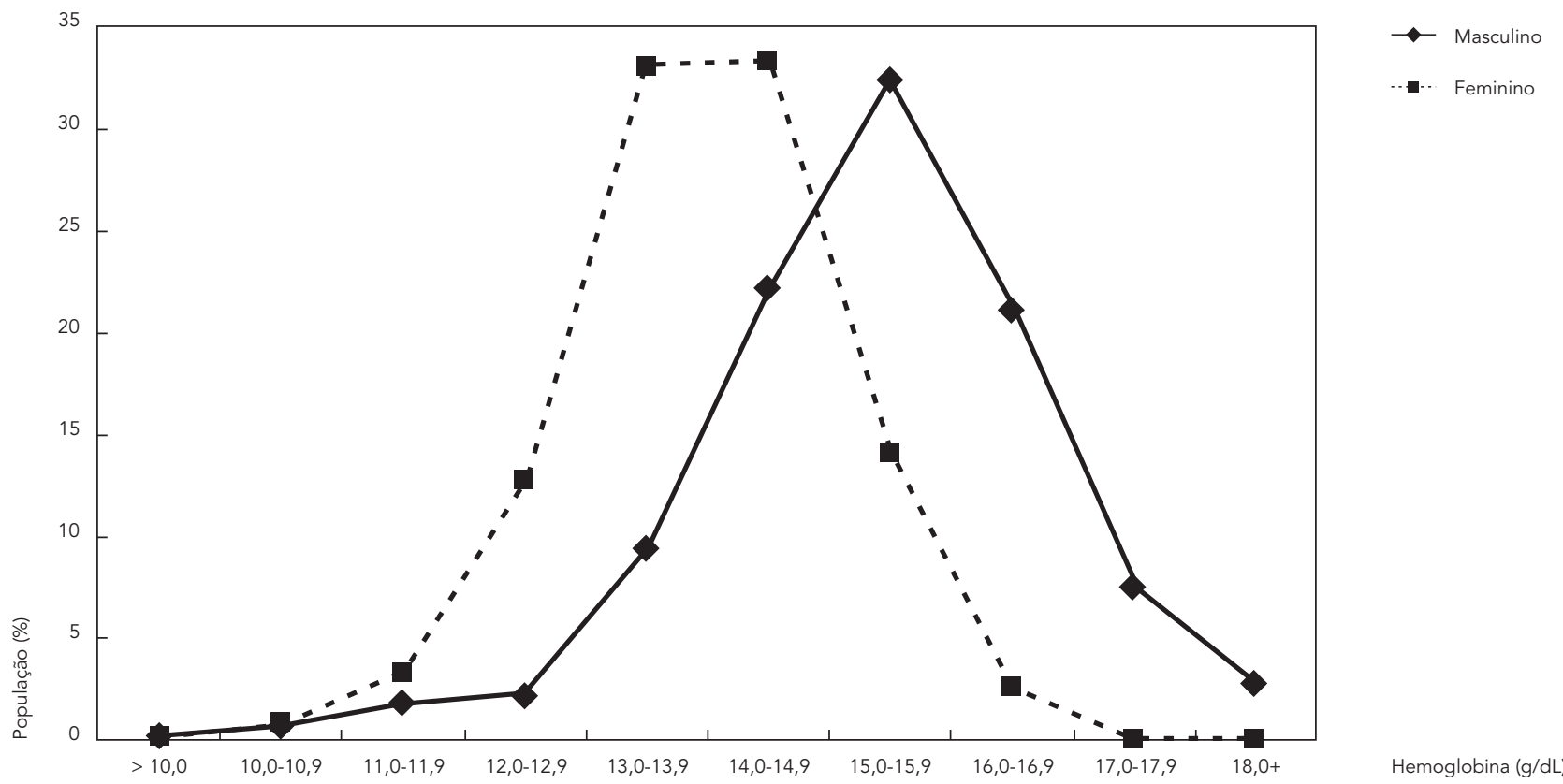


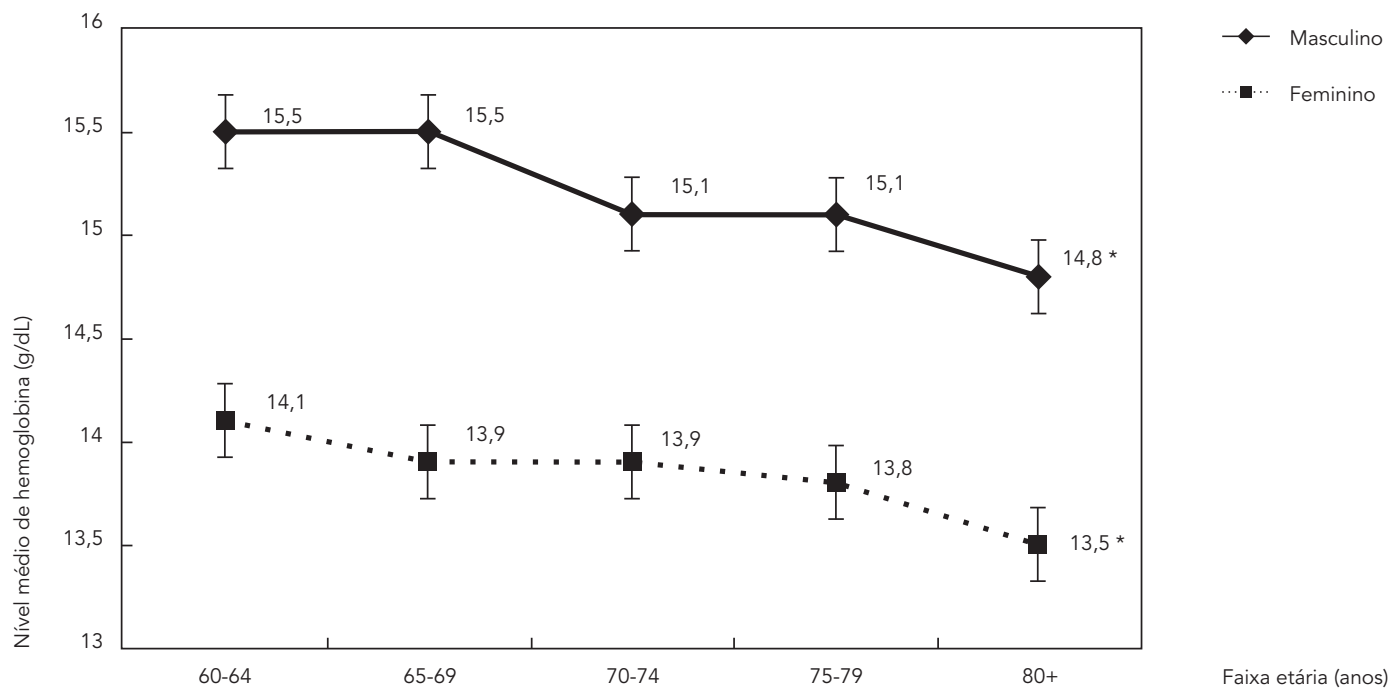

* Diferença significativa $(p<0,05)$, em comparação com os grupos 60-64 e 65-69.

\section{Tabela 2}

Nível médio de hemoglobina segundo indicadores do uso de serviços de saúde e estado nutricional entre homens e mulheres. Projeto Bambuí, Minas Gerais, Brasil, 1997.

\begin{tabular}{|c|c|c|}
\hline \multirow[t]{2}{*}{ Variáveis } & \multicolumn{2}{|c|}{ Nível de hemoglobina (g/dL) média (desvio-padrão) } \\
\hline & Homens $(n=561)$ & Mulheres $(n=880)$ \\
\hline \multicolumn{3}{|c|}{ Hospitalizações nos últimos 12 meses } \\
\hline $\operatorname{Sim}$ & $15,33(1,44)$ & $13,96(1,06)$ \\
\hline \multirow[t]{2}{*}{ Não } & $15,12(1,43)$ & $13,76(1,15)$ \\
\hline & $p=0,09$ & $p=0,01$ \\
\hline \multicolumn{3}{|c|}{ Número de consultas médicas nos últimos 12 meses } \\
\hline 0 & $15,53(1,37)$ a & $14,11(0,99)$ \\
\hline $1-3$ & $15,26(1,34) a b$ & $13,91(1,14)$ \\
\hline \multirow[t]{2}{*}{$\geq 4$} & $15,06(1,72) b$ & $13,84(1,02)$ \\
\hline & $p=0,02$ & $p=0,07$ \\
\hline \multicolumn{3}{|l|}{ Dosagem de albumina } \\
\hline Normal & $15,32(1,42)$ & $13,95(1,06)$ \\
\hline \multirow[t]{2}{*}{ Baixo } & $14,21(2,07)$ & $12,87(1,38)$ \\
\hline & $p=0,002$ & $p<0,0001$ \\
\hline \multicolumn{3}{|l|}{ Índice de massa corporal } \\
\hline Desnutrição & $14,72(1,41)$ a & $13,91(1,05)$ \\
\hline Eutrofia & $15,17(1,34)$ a & $13,79(1,15)$ \\
\hline \multirow[t]{2}{*}{ Sobrepeso/Obesidade } & $15,62(1,53) b$ & $13,98(1,02)$ \\
\hline & $p<0,0001$ & $p=0,315$ \\
\hline
\end{tabular}

Valor de p: teste t de Student ou ANOVA complementada com Bonferroni.

Nota: As letras a e b são referentes às diferenças estatísticas observadas no teste ANOVA/Bonferroni. 
Indicadores de uso de serviços de saúde e do estado nutricional associados ao nível de hemoglobina (g/dL) entre idosos, segundo sexo. Projeto Bambuí, Minas Gerais, Brasil, 1997.

\begin{tabular}{|c|c|c|c|c|}
\hline \multirow[t]{2}{*}{ Variáveis } & \multicolumn{2}{|c|}{ Homens } & \multicolumn{2}{|c|}{ Mulheres } \\
\hline & $\beta$ & IC95\% & $\beta$ & IC95\% \\
\hline \multicolumn{5}{|l|}{ Número de consultas médicas nos últimos 12 meses (vs. nenhuma) } \\
\hline $1-3$ & $-0,24$ & $-0,53 ; 0,05$ & $-0,15$ & $-0,37 ; 0,06$ \\
\hline$\geq 4$ & $-0,42$ & $-0,80 ;-0,04$ & $-0,24$ & $-0,49 ;-0,003$ \\
\hline Ocorrência de hospitalizações nos últimos 12 meses (vs. nenhuma) & 0,02 & $-0,32 ; 0,35$ & $-0,04$ & $-0,21 ; 0,13$ \\
\hline Índice de massa corporal $\left(\mathrm{kg} / \mathrm{m}^{2}\right)$ & 0,07 & 0,$04 ; 0,10$ & 0,02 & 0,$004 ; 0,03$ \\
\hline Dosagem de albumina (mg/dL) & 0,40 & 0,$16 ; 0,62$ & 0,40 & 0,$26 ; 0,53$ \\
\hline
\end{tabular}

IC95\%: intervalo de 95\% de confiança.

mais consultas médicas nos últimos 12 meses foi associado com menores níveis de hemoglobina ( $\beta=-0,42$ para homens e $\beta=-0,24$ para mulheres). O aumento do IMC ( $\beta=0,07$ para homens e $\beta=0,02$ para mulheres) e da dosagem de albumina ( $\beta=0,40$ para ambos os sexos) apresentou associação significativa com maiores níveis de $\mathrm{Hb}$ em ambos os sexos. Não houve associação entre nível de Hb e ocorrência de hospitalizações na população estudada.

\section{Discussão}

A redução dos níveis de $\mathrm{Hb}$ e a ocorrência de anemia em idosos já foram consideradas como consequência natural do processo de envelhecimento 6,29 . No entanto, evidências científicas acumuladas ao longo do tempo demonstraram que a anemia está relacionada a piores condições de saúde, aumentando a vulnerabilidade dessa população para consequências adversas 6,8 . A prevalência de anemia na população idosa de Bambuí foi de 4,5\%, não apresentando diferença significativa entre os sexos. Esse percentual é superior àquele esperado para uma população saudável (2,5\%), mas não se configura como um problema de saúde pública $(<5 \%) 22$.

Diversas pesquisas realizadas no Brasil e em outros países mostraram resultados contrastantes no que se refere à prevalência de anemia, considerando os mesmos pontos de corte adotados no presente estudo. Entre idosos cadastrados no PSF de dois municípios brasileiros, a prevalência de anemia em Viçosa, Minas Gerais (4,5\%)13, foi semelhante à observada em Bambuí; contudo, entre os idosos do Município de Camaragibe, Pernambuco 14, essa prevalência foi mais elevada (12\%). Em amostra não probabilística de idosos saudáveis do Município de Gravataí 1, Rio Grande do Sul, a anemia foi diagnosticada em 4,3\% da população. Em estudo de base populacional conduzido em áreas de baixa condição socioeconômica do Município de São Paulo 15, a prevalência de anemia entre indivíduos com idade igual ou superior a 65 anos foi também mais elevada $(10,2 \%)$, quando comparada à observada em Bambuí. Investigações realizadas em outros países também encontraram prevalências mais elevadas, com exceção da Coreia do Sul 30, com apenas 1,5\% de idosos anêmicos. Nos Estados Unidos 6, dados do National Health and Nutrition Examination Survey (NHANES III) demonstraram que $10,6 \%$ dos indivíduos com 65 anos ou mais estavam anêmicos, enquanto, entre os idosos participantes de outro estudo americano (Cardiovascular Health Study - CHS) 31, a prevalência foi de 8,5\%. Na Itália, o InCHIANTY Study 32 também encontrou prevalência superior (12\%) à observada em Bambuí.

Apesar da grande variação na prevalência de anemia observada na literatura, os resultados são consistentes no que se refere à redução dos níveis de $\mathrm{Hb}$ com o aumento da idade, elevando a prevalência de anemia entre os idosos mais velhos 30,32,33, como observado na população de Bambuí. Dois estudos norte-americanos de base populacional ilustram esse fato. Em um deles 34, a prevalência de anemia aumentou significativamente com o aumento da idade, verificandose que $32 \%$ dos idosos acima de 80 anos tinham anemia, em comparação a $25 \%$ daqueles entre 75 e 79 anos e $17 \%$ dos com idade entre 65 e 74 anos. Da mesma forma, idosos acima de 85 anos avaliados no NHANES III 6 apresentaram prevalência de anemia igual a $25 \%$, expressivamente maior que a encontrada na população idosa total (10,6\%). Dados do InCHIANTY Study 35 
indicaram que, em média, há um declínio de 0,75g/dL nos níveis de $\mathrm{Hb}$ em homens e de 0,5g/ $\mathrm{dL}$ em mulheres após os 60 anos. Esse achado justifica a relação entre a prevalência de anemia e a idade do indivíduo, ressaltando a importância dos baixos níveis de $\mathrm{Hb}$ para a saúde de idosos mais velhos.

A associação entre baixos níveis de Hb e pior estado nutricional não tem sido muito explorada na literatura 17,18 , sobretudo em estudos de base populacional, apesar de a deficiência nutricional ser considerada um importante fator etiológico para anemia 2. Por outro lado, entre idosos hospitalizados essa relação já está bem estabelecida, sendo a anemia fortemente associada a baixos valores de IMC e de albumina sérica 8,18,19,20. Chama a atenção o fato de que os resultados encontrados em Bambuí são congruentes com os estudos de base populacional realizados em outros países, reforçando a associação encontrada na literatura para população idosa hospitalizada. Dados da linha de base do estudo prospectivo norte-americano Established Populations for Epidemiologic Studies of the Elderly 24 demonstraram que a anemia ou anemia limítrofe (Hb entre 12 e 13g/dL para mulheres e 13 e $14 \mathrm{~g} / \mathrm{dL}$ para homens) apresentou associação significativa com menores valores de IMC e de albumina entre idosos acima de 70 anos vivendo em comunidade. Em estudo realizado com 2.550 adultos de meia idade e idosos chineses $(\geq$ 55 anos) 36, a redução dos níveis de Hb foi associada à também redução dos níveis de albumina sérica. Além disso, aqueles com IMC abaixo do recomendado apresentaram níveis médios de $\mathrm{Hb}$ significativamente menores quando comparados aos com estado nutricional adequado. De forma semelhante, Zakai et al. 31 avaliaram 5.797 idosos de ambos os sexos participantes do Cardiovascular Health Study (Estados Unidos) e verificaram que baixos níveis de $\mathrm{Hb}$ foram significativamente associados a menores valores de IMC e albumina sérica, além de maior fragilidade, insuficiência cardíaca congestiva, derrame e elevados níveis de creatinina. Portanto, os resultados obtidos em Bambuí mostram que essa associação pode ser também verificada entre idosos brasileiros, com destaque para o impacto da deficiência nutricional sobre níveis séricos de Hb nessa população. Em adição, deve ser considerado que essa análise foi ajustada por outros fatores determinantes do nível de hemoglobina em população idosa.

A associação entre anemia e maior utilização dos serviços de saúde pelos idosos está bem estabelecida na literatura, ocasionando aumento importante para os custos do sistema 37,38 . A análise dos dados de idosos cadastrados no Sis- tema de Saúde Integrado dos Estados Unidos 39 verificou associação significativa entre anemia crônica e maior procura pelo serviço de saúde, internações mais prolongadas e, consequentemente, maior custo para o sistema. Outro estudo realizado no mesmo país 40 mostrou que os idosos ( $\geq 65$ anos) anêmicos tinham médias significativamente maiores de consultas médicas e hospitalizações, bem como maior tempo médio de permanência no hospital, quando comparados aos não anêmicos. Maior ocorrência e maior tempo de hospitalizações entre idosos anêmicos, comparados aos não anêmicos, foram também observados em dois estudos prospectivos, um norte-americano 41 e outro italiano ${ }^{10}$. No Brasil, a anemia é relatada, na maioria das vezes, como causa intermediária de internações entre idosos. Estudo realizado com aqueles atendidos em unidades básicas de saúde do Município de Viçosa 42 demonstrou que apenas $1,1 \%$ dos atendimentos prestados a este grupo teve como causa direta a anemia.

Deve-se ressaltar que, além da anemia, baixos níveis de $\mathrm{Hb}$ também têm impacto sobre o aumento do tempo de permanência no ambiente hospitalar e sobre o custo financeiro do tratamento ${ }^{43}$. Sua redução pode representar um primeiro sinal de doenças ainda não diagnosticadas, além de, por si só, estar associada à maior fragilidade do idoso, levando ao aumento da demanda por atendimento em saúde 16,39,40. Em Bambuí, os resultados encontrados são concordantes com os dados expostos. Apesar de o relato de hospitalizações no último ano não ter sido relacionado a menores níveis de $\mathrm{Hb}$, a associação destes com o número de consultas médicas, independentemente de outros fatores de confusão, indica que a relação entre nível de $\mathrm{Hb}$ e uso de serviços de saúde pode também ser observada na população idosa, como forma de primeiro acesso e aumento das demandas de atendimento em saúde ainda numa fase pré-clínica da anemia. Essa associação é relevante para saúde pública, considerando-se o aumento da população idosa observado no Brasil e no mundo, a redução dos níveis de $\mathrm{Hb}$ com o aumento da idade e o aumento dos gastos públicos com o setor saúde associados a essa condição 38,44 .

A característica transversal deste estudo não permite estabelecer uma relação causal entre estado nutricional, consultas médicas e nível de $\mathrm{Hb}$ na população estudada. No entanto, os resultados encontrados contribuem para maior esclarecimento sobre a associação de níveis de $\mathrm{Hb}$ com variáveis nutricionais e uso de serviços de saúde, ressaltando a escassez de estudos de base populacional, sendo este o primeiro realizado no Brasil. 
Concluindo, as associações observadas sugerem um ciclo complexo entre baixos níveis de hemoglobina, desnutrição e uso de serviços de saúde entre idosos, de forma independente do diagnóstico de anemia. Tal relação deve ser considerada para a estruturação de programas de saúde para esse grupo etário, visto que a redução dos níveis de Hb é uma condição potencialmente reversível, e seu tratamento pode adiar ou mesmo prevenir a ocorrência de doenças relacionadas ao envelhecimento.

\section{Resumo}

O objetivo deste estudo foi descrever a prevalência de anemia e avaliar a associação de nível de hemoglobi$n a(\mathrm{Hb})$ com indicadores do estado nutricional e uso de serviços de saúde em uma população idosa $(\geq 60$ anos). Dos 1.742 idosos participantes da linha de base da coorte de Bambuí, Minas Gerais, Brasil, foram incluídos 1.441 (82,7\%). A variável dependente foi o $\mathrm{ni}$ vel de Hb e as independentes foram número de hospitalizações e visitas ao médico nos 12 meses precedentes à entrevista, índice de massa corporal (IMC) e albumi na sérica. Foi realizada regressão linear multivariada estratificada por sexo e ajustada por fatores de confusão. A prevalência de anemia foi baixa na população (4,5\%), sem diferenças entre sexos e significativamente maior em idosos mais velhos (10,2\%). Baixos níveis de $\mathrm{Hb}$ foram associados ao maior número de consultas médicas e a menores valores de albumina sérica e IMC em ambos os sexos. O estudo sugere um ciclo complexo entre baixos níveis de $\mathrm{Hb}$, desnutrição e atendimento médico, o que deve ser considerado na estruturação de programas de saúde para idosos.

Envelhecimento; Hemoglobinas; Anemia; Estado Nutricional; Serviços de Saúde

\section{Colaboradores}

C. L. A. Silva realizou a análise dos dados, a interpretação dos resultados e a redação do manuscrito. M. F Lima-Costa e S. V. Peixoto orientaram o trabalho, participando da discussão dos resultados, redação e revisão do texto final. J. O. A. Firmo coordenou o trabalho de campo de Bambuí e participou da discussão dos resultados e revisão do texto. 


\section{Referências}

1. Schaan MDA, Schwanke CHA, Bauer M, Luz C, Cruz IM. Hematological and nutritional parameters in apparently healthy elderly individuals. Rev Bras Hematol Hemoter 2007; 29:136-43.

2. Patel KV. Variability and heritability of hemoglobin concentration: an opportunity to improve understanding of anemia in older adults. Haematologica 2008; 93:1281-3.

3. Eisenstaedt R, Penninx BWJH, Woodman RC. Anemia in the elderly: current understanding and emerging concepts. Blood Rev 2006; 20:213-26.

4. Price EA. Aging and erythropoiesis: current state of knowledge. Blood Cells Mol Dis 2008; 41:158-65.

5. Balducci L. Epidemiology of anemia in the elderly: information on diagnostic evaluation. J Am Geriatr Soc 2003; 51(3 Suppl):2-9.

6. Guralnik JM, Einstaedt RS, Ferruci L. Prevalence of anemia in persons 65 years and older in the United States: evidence for a high rate of unexplained anemia. Blood 2004; 104:2263-88.

7. Lippi G, Franchini M, Salvagno GL, Montagnana M, Targher G, Guidi GG. Determinants of anemia in the very elderly: a major contribution from impaired renal function? Blood Transfus 2010; 8:44-8.

8. Price EA, Mehra R, Holmes TH, Schrier SL. Anemia in older persons: etiology and evaluation. Blood Cells Mol Dis 2011; 46:159-65.

9. Culleton BF, Manns BJ, Zhang J, Tonelli M, Klarenbach S, Hemmelgam BR. Impact of anemia on hospitalization and mortality in older adults. Blood 2006; 107:3841-6.

10. Riva E, Tettamanti M, Mosconi P, Apolone G, Gandini F, Nobili A, et al. Association of mild anemia with hospitalization and mortality in the elderly: the Health and Anemia Population-Based Study. Haematologica 2009; 94:22-8.

11. Lucca U, Tettamanti M, Mosconi P, Apolone G, Gandini F, Nobili A, et al. Association of mild anemia with cognitive, functional, mood and quality of life outcomes in the elderly: the "Health and Anemia" Study. PLoS One 2008; 3:e1920.

12. Tettamanti M, Lucca U, Gandini F, Recchia A, Mosconi P, Apolone G, et al. Prevalence, incidence and types of mild anemia in the elderly: the "Health and Anemia" Population-Based Study. Haematologica 2010; 95:1849-56.

13. Silva CLA. Fatores associados ao estado nutricional e ao nível de hemoglobina em idosos: Programa de Saúde da Família, Viçosa-MG [Dissertação de Mestrado]. Viçosa: Universidade Federal de Viçosa; 2008.

14. Barbosa DL, Arruda HKG, Diniz AS. Prevalência e caracterização da anemia em idosos do Programa de Saúde da Família. Rev Bras Hematol Hemoter 2006; 28:288-92.

15. Santos IS, Scazufca M, Lotufo PA, Menezes PR, Benseñor IM. Anemia and dementia among the elderly: the São Paulo Ageing \& Health Study. Int Psychogeriatr 2012; 24:74-81.
16. Balducci L, Aapro M. Anemia and aging or anemia of aging? In: Balducci L, Ershler WB, Bennett JM, editors. Anemia in the elderly. New York: Springer; 2008. p. 21-38.

17. Guralnik JM, Ershler WB, Schirier SL, Picozzi VJ. Anemia in the elderly: a public health crisis in hematology. Hematology Am Soc Hematol Educ Program 2005:528-32.

18. Mitrache C, Passweg JR, Libura J, Petrikkos L, Seiler WO, Gratwohl A, et al. Anemia: an indicator for malnutrition in the elderly. Ann Hematol 2001 80:295-8.

19. Horwich TB, Fonarow GC, Hamilton MA, Maclellan WR, Borenstein J. Anemia is associated with worse symptoms, greater impairment in functional capacity and a significant increase in mortality in patients with advanced heart failure. J Am Coll Cardiol 2002; 39:1780-6.

20. Ramel A, Jonsson PV, Bjornsson S, Thorsdottir I. Anemia, nutritional status, and inflamation in hospitalized elderly. Nutrition 2008; 24:1116-22.

21. Lima-Costa MF, Firmo JOA, Uchôa E. The Bambuí Cohort Study of Aging: methodology and health profile of participants at baseline. Cad Saúde Pública 2011; 27 Suppl 3:S327-35.

22. World Health Organization. Iron deficiency anaemia. Assessment, prevention, and control. Geneva: World Healht Oraganization; 2001.

23. World Health Organization. Physical status: the use and interpretation of anthropometry. Report of a WHO committee. Geneva: World Health Organization; 1998.

24. Penninx BWJH, Guralnik JM, Onder G, Ferruci L, Wallace RB, Pahor M. Anemia and decline in physical performance among older persons. Am J Med 2003; 113:104-10.

25. Penninx BWJH, Pahor M, Cesari M, Corsi AM, Woodman RC, Bandinelli S, et al. Anemia is associated with disability and decreased physical performance and muscle strength in the elderly. J Am Geriatr Soc 2004; 52:719-24.

26. Shi Z, Hu X, Yuan B, Pan X, Meyer HE, HolboeOttensen G. Association between serum ferritin, hemoglobin, iron intake, and diabetes in adults in Jiangsu, China. Diabetes Care 2006; 29:1878-83.

27. Chaves PHM. Functional outcomes of anemia in older adults. Semin Hematol 2008; 45:255-60.

28. Lee YT, Chiu HC, Su HM, Yang JF, Voon WC, Lin TH, et al. Lower hemoglobin concentrations and subsequent decline in kidney function in an apparently healthy population aged 60 year and older. Clin Chim Acta 2008; 389:25-30.

29. Izaks GJ, Westendorp RGJ, Kook DL. The definition of anemia in older persons. JAMA 1999 281:1714-7.

30. Choi CH, Lee J, Park KH, Choi IK, Kim SJ, Seo JH, et al. Incidence of anemia in older Koreans: community-based cohort study. Arch Gerontol Geriatr $2005 ; 41: 303-9$ 
31. Zakai NA, Katz R, Hirsch C, Shlipak MG, Chaves PHM, Newman AB, et al. A prospective study of anemia status, hemoglobin concentration, and mortality in a elderly cohort. Arch Intern Med 2005; 165:2214-20.

32. Ble A, Fink JC, Woodman RC, Klausner MA, Guralnik JM, Ferruci L. Renal function, erythropoietin, and anemia of older persons: the InCHIANTI study. Arch Inter Med 2005; 165:2222-7.

33. Beghé C, Wilson A, Ershler WB. Prevalence and outcomes of anemia en geriatrics: a systematic review of literature. Am J Med 2004; 116 Suppl 7 : S3-10.

34. Denny SD, Kuchibhatla MN, Cohen HJ. Impact of anemia on mmortality, cognition, and function in community-dwelling elderly. Am J Med 2006; 119:327-34.

35. Adamson JW. Renal disease and anemia in the elderly. Semin Hematol 2008; 45:235-41.

36. Te-Pin NG, Feng L, Niti M, Yap KB. Albumin, haemoglobin, BMI and cognitive performance in older adults. Age Ageing 2008; 37:423-9.

37. Gualandro SFM, Hojaij NHSL, Jacob Filho W. Deficiência de ferro no idoso. Rev Bras Hematol Hemoter 2010; 32 Suppl 2:57-61.

38. Smith RE. The clinical and economic burden of anemia. Am J Manag Care 2010; 16 Suppl:S59-66.
39. Carmel R. Nutritional anemias and the elderly. Semin Hematol 2008; 45:225-34.

40. Chaves PHM, Mody SH, Blasi MV, Siegartel LR, Stern LS, Doyle JJ, et al. Healthcare costs and resource utilization associated with chronic anemia in older adults. J Manag Care Med 2005; 8:13-20.

41. Penninx BW, Pahor M, Woodman RC, Guralnik JM. Anemia in old age is associated with increased mortality and hospitalization. J Gerontol A Biol Sci Med Sci 2006; 61:474-9.

42. Venturi I, Rosado LEFPL, Cotta RMM, Rosado GP, Doimo LA, Tinoco ALA, et al. Identificação da área de influência do serviço de atenção básica do sistema público de saúde à população idosa, município de Viçosa - MG. Ciênc Saúde Coletiva 2008; 13:1293-304.

43. Nunes A. O envelhecimento populacional e as despesas do sistema único de saúde. In: Camarano AA, organizador. Novos idosos brasileiros: muito além dos 60? Rio de Janeiro: Instituto de Pesquisa Econômica Aplicada; 2004. p. 427-53.

44. Robinson B. Cost of anemia in the elderly. J Am Geriatr Soc 2003; 51 Suppl:S14-7.

Recebido em 26/Mar/2012

Aprovado em 17/Ago/2012 